\title{
The divergence of Banach space valued random variables on Wiener space
}

\author{
E. Mayer-Wolf • M. Zakai
}

Published online: 20 November 2007

(C) Springer-Verlag 2007

\section{Erratum to: Probab. Theory Relat. Fields (2005) 132:291-320 DOI 10.1007/s00440-004-0397-0}

The original version of this article unfortunately contained two mistakes.

Corollary 3.5 (as well as Corollary 3.17 a whose proof relies on it) should be ignored since the inequality $\left|F_{n}\right|_{p, 1} \leq\left\|F_{n}\right\|_{p, 1}$ in its proof is false, and we have been unable to find a simple alternative argument.

More importantly, in Propositions 3.14 and 3.18 one needs to add the assumption

(A) $Y^{* *}$ has the Radon Nykodim property (RNP) with respect to $\mu$ (cf. [1])

on the Banach space $Y$, as we shall now explain. Note (Sect. III.3 in [1]) that $Y^{* *}$ has the RNP with respect to any measure if, for example, $Y^{* *}$ is separable or $Y$ is reflexive.

Unfortunately, in the proof of Proposition 3.14, the natural imbedding of $L^{p}\left(\mu ; Y^{* *}\right)$ in the operator space $L\left(Y^{*}, L^{p}(\mu)\right)$ was erroneously claimed to be surjective. In addition, the observation associated with (3.12) was also incorrect as stated (although not used in the rest of the paper). We restate this observation in (1) below, and prove it under the additional assumption (A); it will then be used in (2) to replace the incorrect proof of Proposition 3.14.

The online version of the original article can be found under doi:10.1007/s00440-004-0397-0.

E. Mayer-Wolf $(\bowtie)$

Department of Mathematics, Technion I.I.T., Haifa, Israel

e-mail: emw@techunix.technion.ac.il

M. Zakai

Department of Electrical Engineering, Technion I.I.T., Haifa, Israel 
(1) Assume (A) and let $\mathbf{K} \in L^{p}\left(\mu ; L\left(W^{*}, Y^{* *}\right)\right.$. If (3.12) holds for some $\gamma>0$ and all $F \in \mathcal{S}\left(Y^{*}\right)$ then $\mathbf{K} \in \mathbf{d o m}_{p, Y^{* *} \boldsymbol{\delta}}$ (the converse is obvious).

Proof The bound (3.12) implies the existence of a $\Lambda_{\mathbf{K}} \in L^{q}\left(\mu, Y^{*}\right)^{*}$ such that

$$
E \operatorname{tr}\left(\mathbf{K}^{T} \nabla^{W^{*}} F\right)=\Lambda_{\mathbf{K}}(F) \quad \forall F \in \mathcal{S}\left(Y^{*}\right)
$$

Due to assumption (A), $L^{q}\left(\mu, Y^{*}\right)^{*}$ can be identified with $L^{p}\left(\mu, Y^{* *}\right)$ (cf. Theorem IV.1 in [1]) in the sense that there exists a $\delta \mathbf{K} \in L^{p}\left(\mu, Y^{* *}\right)$ such that $\Lambda_{\mathbf{K}}(F)$ is given by $E_{Y^{*}}\langle F, \delta \mathbf{K}\rangle_{Y^{* *}}$. Thus, for any $F \in \mathcal{S}\left(Y^{*}\right)$

$$
E \operatorname{tr}\left(\mathbf{K}^{T} \nabla^{W^{*}} F\right)=E_{Y^{* *}}\langle\boldsymbol{\delta} \mathbf{K}, F\rangle_{Y^{* * *}} \cdot
$$

For $F=\phi\left(\delta\left(e_{1}\right), \ldots, \delta\left(e_{m}\right)\right) \otimes l, \quad\left(\left\{e_{1}, \ldots, e_{m}\right\} \subset W^{*}\right.$, orthonormal in $H$, and $\left.l \in Y^{*}\right), \quad(\dagger)$ amounts to ${ }_{Y^{* *}}\left\langle E\left(\sum_{i=1}^{m} \partial_{i} \phi K e_{i}\right), l\right\rangle_{Y^{* * *}}={ }_{Y^{* *}}\langle E \phi \delta \mathbf{K}, l\rangle_{Y^{* * *}}$ which, if true $\forall l \in Y^{*}$, is true $\forall l \in Y^{* * *}$ as well. Thus ( $\dagger$ ) holds for all $F \in \mathcal{S}\left(Y^{* * *}\right)$,

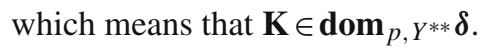

(2) We now present a modified proof of the "if" implication in the first statement of Proposition 3.14, using the characterization provided by (1) instead of the erroneous identification of $L^{p}\left(\mu ; Y^{* *}\right)$ and $L\left(Y^{*}, L^{p}(\mu)\right)$ mentioned above:

It follows from (3.13) that there exists a $\Delta_{\mathbf{K}} \in L\left(Y^{*}, L^{p}(\mu)\right)$ such that for all $l \in Y^{*}$

$$
\delta\left(\mathbf{K}^{T} l\right)=\Delta_{\mathbf{K}}(l)
$$

so that, for any $F=\sum_{j=1}^{m} \Phi_{j} l_{j} \in \mathcal{S}\left(Y^{*}\right)$

$$
\begin{aligned}
E \operatorname{tr}\left(\mathbf{K}^{T} \nabla F\right) & =E \sum_{j=1}^{m} \operatorname{tr} \mathbf{K}^{T} \nabla\left(\Phi_{j} l_{j}\right)=\sum_{j=1}^{m} E_{W^{*}}\left\langle\nabla \Phi_{j}, \mathbf{K}^{T} l_{j}\right\rangle_{W^{* *}} \\
& =\sum_{j=1}^{m} E \delta\left(\mathbf{K}^{T} l_{j}\right) \Phi_{j}=\sum_{j=1}^{m} E \Delta_{\mathbf{K}}\left(l_{j}\right) \Phi_{j} \\
& =E \Delta_{\mathbf{K}}\left(\sum_{j=1}^{m} \Phi_{j} l_{j}\right)=E \Delta_{\mathbf{K}}(F)
\end{aligned}
$$

and thus for any $q \geq 1$, and with $\left\|\Delta_{\mathbf{K}}\right\|$ denoting the operator norm,

$$
\left|E \operatorname{tr}\left(\mathbf{K}^{T} \nabla F\right)\right| \leq E\left\|\Delta_{\mathbf{K}}\right\|\|F\|_{Y^{*}} \leq\left\|\Delta_{\mathbf{K}}\right\|\left(E\|F\|_{Y^{*}}^{q}\right)^{1 / q}
$$

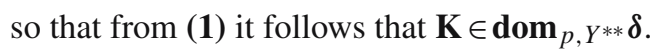


Acknowledgements We are grateful to J. Maas and J. Van Neerven for drawing our attention to the two mistakes addressed here.

\section{Reference}

1. Diestel, J., Uhl, J.J., Jr: Vector measures. AMS Math. Surv. 15, (1977) 